\title{
Amplitude control of a quantum state in non-Hermitian Rice-Mele model driven by an external field
}

\author{
S. Lin $^{1}$, X. Z. Zhang, ${ }^{2}$ and Z. Song田 \\ ${ }^{1}$ School of Physics, Nankai University, Tianjin 300071, China \\ ${ }^{2}$ College of Physics and Materials Science, Tianjin Normal University, Tianjin 300387, China
}

\begin{abstract}
In the Hermitian regime, the Berry phase is always a real number. It may be imaginary for a non-Hermitian system, which leads to amplitude amplification or attenuation of an evolved quantum state. We study the dynamics of the non-Hermitian Rice-Mele model driven by a time-dependent external field. The exact results show that it can have full real spectrum for any value of the field. Several rigorous results are presented for the Berry phase with respect to the varying field. We demonstrate that the Berry phase is the same complex constant for any initial state in a single subband. Numerical simulation indicates that the amplitude control of a state can be accomplished by a quasi-adiabatic process within a short time.
\end{abstract}

PACS numbers: 11.30.Er, 03.65.Vf, 03.65.-w

\section{INTRODUCTION}

In the Hermitian regime, the Berry phase [1-3] is always the real number. It may be complex for a nonHermitian system, the concept of which was first introduced by Garrison and Wright [4] in a dissipative system. And in these years the geometric phase for quantum systems governed by non-Hermitian Hamiltonians and complex-valued geometric phase effects have gained considerable attention and have been studied by various authors [5-17]. The existence of imagine part of the phase could lead to amplitude amplification or attenuation of an evolved quantum state. We investigate the dynamics of the non-Hermitian Rice-Mele model driven by a time-dependent external field. The exact results show that it can have full real spectrum for any value of the field and several rigorous results are presented for the Berry phase with respect to the varying field. We find that the Berry phase is the same complex constant for any initial state in a single sub-band. And via numerical simulations, the amplitude control of a state can be achieved by a quasi-adiabatic process within a short time.

In a Hermitian quantum system, the geometric phase acquired during an adiabatic evolution is always real and can bring nothing to an evolved quantum state if only one eigenstate is involved. It has been shown that a non-Hermitian system can have real spectrum [18] and possess peculiar phenomena. These include fast propagation [19], infinite reflection coefficient 20 22], unidirectional transmission [23], transmission phase lapse [24], maximum multi-particle entanglement associated with the phase transition [25] as well as the complex Berry phase. The imaginary part of the Berry phase is significant for a propagating particle since it may be utilized to directly amplify or attenuate the particle probability. Very recently, the spectral and dynamical properties of

\footnotetext{
* songtc@nankai.edu.cn
}

a quantum particle constrained on a ring threaded by a time-varying magnetic flux in the presence of a complex (non-Hermitian) potential are investigated [26]. It has been demonstrated that several striking effects are observed in the non-Hermitian case in comparison with the Hermitian one.

In a previous work [27], the dynamical behavior has been investigated for a non-Hermitian Rice-Mele model in the absence of an external magnetic field. It has been shown that within the unbroken $\mathcal{P} \mathcal{T}$-symmetric region, the translational symmetry ensures the probabilitypreserving evolution of a state, which involves only one sub-band or two sub-bands with different $k$. In this paper we aim at investigating the dynamical behaviors in the same model but in the presence of a time-varying flux. We have determined that the law of probability preservation still holds in the presence of a constant flux. When the flux changes adiabatically, we will show rigorously that the Berry phases of all the eigenstates within a sub-band are identical complex numbers, which depend on the combination of the system parameters, including the lattice distortion, imaginary potential, and flux. The imaginary Berry phase leads to the amplification and attenuation of the amplitude of an evolved quantum state. In contrast to the review [28] considered in the situation where the Hamiltonian has singularities, i.e., diabolic or exceptional points, and all the Berry phases in the review are encircling these special points, we would like to emphasize the phases investigated in our paper are not involving these special points. We also provide some illustrative simulations to show that the amplitude control of a wave packet can be accomplished by a quasi-adiabatic process within a short time.

This paper is organized as follows. In Sec. II we introduce the non-Hermitian Rice-Mele model and its exact solution. Section [II] is dedicated to show how the amplitude modulation is determined by the imaginary Berry phase under the time-dependent Hamiltonian. In Sec. IV] we investigate wave packet dynamics with its accomplishment of amplitude control by a quasi-adiabatic 


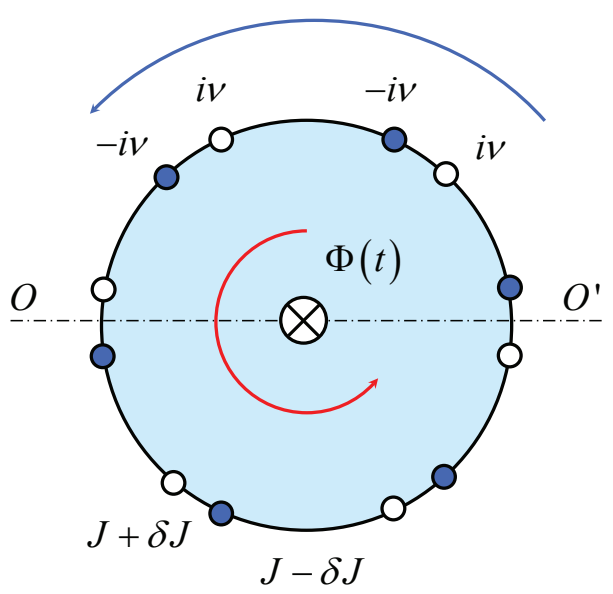

FIG. 1. (Color online) Schematic illustration of the nonHermitian Rice-Mele model driven by a time-dependent external field. It is $\mathcal{P} \mathcal{T}$-symmetric with respect to the $O O^{\prime}$ axis in the absence of the field. Constant field $\Phi$ breaks the $\mathcal{P} \mathcal{T}$-symmetry, but keeps the translational symmetry. A timevarying field $\Phi(t)$ induces eddy field in a direction indicated by the red arrow. Together with the distortion, the imaginary potentials can also break the left-right chiral symmetry, inducing a direction of the system indicated by the blue arrow. In the case that two arrows are either the same or the opposite, the dynamics of a state exhibits different behaviors.

process within a relatively short time. Finally, we give a summary and discussion in Sec. V

\section{MODEL AND SOLUTIONS}

We consider a non-Hermitian Rice-Mele model 29] with a flux, which can be described by the following Hamiltonian

$$
\begin{aligned}
H= & -J \sum_{j=1}^{2 N}\left[1+(-1)^{j} \delta\right]\left(e^{i \phi} c_{j}^{\dagger} c_{j+1}+\text { H.c. }\right) \\
& +J(\mu+i \nu) \sum_{j}(-1)^{j} c_{j}^{\dagger} c_{j}
\end{aligned}
$$

on a $2 N$-site lattice, where $c_{j}^{\dagger}$ is the creation operator of a boson (or a fermion) at the $j$ th site with the periodic boundary condition $c_{2 N+1}=c_{1}$. Here the hopping amplitude is modulated by the dimerization factor $\delta$, and $\Phi=2 N \phi$ is the magnetic flux threading the ring. Staggered complex potential induces the non-Hermiticity of the model.

There are three elements in the structure of the model, lattice distortion, imaginary potentials, and flux. As is shown in Fig. 1, the imaginary potentials and distortion can break the left-right chiral symmetry [27]. In addition, a time-varying field $\Phi(t)$ induces eddy field in another direction. The dynamics of a state should exhibit different behaviors with different configurations. It turns out that imaginary potentials can appear in open physical systems 30 34]. In experiments, the effective magnetic flux threading a ring can be realized by rotating the lattice 35, [36].

In the absence of the imaginary potential and flux, this model has been adequately studied in various perspectives [3]. In the case of purely imaginary potential and $\phi=0$, the model has $\mathcal{P} \mathcal{T}$ symmetry, and the dynamics has been systematically investigated [27] in the frameworks of biorthogonal and Dirac inner products. In this paper, we refer particle probability to Dirac probability. Moreover, we introduce the magnetic flux to the Rice-Mele model, which has been employed to control the dynamics of wave packet [37 39]. Although non-zero $\phi$ breaks the $\mathcal{P} \mathcal{T}$ symmetry, we will show that this model can have full real spectrum. We note that the Hamiltonian is invariant via a translational transformation, i.e., $\left[T_{2}, H\right]=0$, where $T_{2}$ is the shift operator that defined as

$$
T_{2}^{-1} c_{i}^{\dagger} T_{2}=c_{i+2}^{\dagger} .
$$

This allows the invariant subspace spanned by the eigenvector of operator $T_{2}$. The single-particle eigenvector of $T_{2}$ can be expressed as $a_{k}^{\dagger}|0\rangle$ and $b_{k}^{\dagger}|0\rangle$, where

$$
\begin{aligned}
& a_{k}^{\dagger}=\frac{1}{\sqrt{N}} \sum_{j} e^{i k j} c_{2 j-1}^{\dagger}, \\
& b_{k}^{\dagger}=\frac{1}{\sqrt{N}} \sum_{j} e^{i k j} c_{2 j}^{\dagger},
\end{aligned}
$$

satisfying

$$
T_{2}^{-1} a_{k}^{\dagger} T_{2}=e^{-i k} a_{k}^{\dagger}, T_{2}^{-1} b_{k}^{\dagger} T_{2}=e^{-i k} b_{k}^{\dagger} .
$$

Here, $a_{k}^{\dagger}$ and $b_{k}^{\dagger}$ are two kinds of creation operators of bosons (or fermions), with $k=2 \pi n / N(n \in[1, N])$, representing the particles in odd and even sublattices. Then the original Hamiltonian $H$ can be expressed as

$$
H=\sum_{k} H_{k},
$$

where

$$
\begin{aligned}
H_{k}= & \Lambda(k, \phi) J a_{k}^{\dagger} b_{k}+\text { H.c. } \\
& -(\mu+i \nu) J\left(a_{k}^{\dagger} a_{k}-b_{k}^{\dagger} b_{k}\right),
\end{aligned}
$$

and

$$
\Lambda(k, \phi)=-e^{-i k / 2} \sum_{\lambda= \pm}(1-\lambda \delta) e^{i \lambda(k / 2+\phi)} .
$$

It is easy to check that

$$
\left[H_{k}, H_{k^{\prime}}\right]=0,
$$

which ensures us to arrive at the solution in each invariant subspace. 
Considering the single-particle solution, we can introduce the pseudo-spin operators

$$
s_{k}^{+}=\left(s_{k}^{-}\right)^{\dagger}=a_{k}^{\dagger} b_{k}, s_{k}^{z}=\frac{1}{2}\left(a_{k}^{\dagger} a_{k}-b_{k}^{\dagger} b_{k}\right),
$$

which obey

$$
\left[s_{k}^{+}, s_{k}^{-}\right]=2 s_{k}^{z},\left[s_{k}^{z}, s_{k}^{ \pm}\right]= \pm s_{k}^{ \pm} .
$$

Accordingly, $H_{k}$ has the form

$$
H_{k}=\vec{B}_{k} \cdot \vec{\sigma}_{k}
$$

where $\vec{\sigma}_{k}$ is Pauli matrix. Components of the field $\vec{B}_{k}$ in the rectangular coordinates are

$$
\begin{aligned}
& B_{k}^{x} / J=-(1-\delta) \cos \phi-(1+\delta) \cos (k+\phi), \\
& B_{k}^{y} / J=(1-\delta) \sin \phi-(1+\delta) \sin (k+\phi), \\
& B_{k}^{z} / J=-(\mu+i \nu)
\end{aligned}
$$

where

$$
\cos \theta_{k}=\frac{B_{k}^{z}}{B_{k}}, \tan \varphi_{k}=\frac{B_{k}^{y}}{B_{k}^{x}}
$$

and the field magnitude is

$$
B_{k}=\left[\left(B_{k}^{x}\right)^{2}+\left(B_{k}^{y}\right)^{2}+\left(B_{k}^{z}\right)^{2}\right]^{1 / 2} .
$$

Obviously, $\theta_{k}$ can be a complex number even in the case with real $B_{k}$.

The eigenvalues of $H_{k}$ are

$$
\begin{aligned}
\varepsilon_{ \pm}^{k}= & \pm B_{k} \\
= & \pm 2 J\left[(\mu+i \nu)^{2} / 4+\delta^{2}\right. \\
& \left.+\left(1-\delta^{2}\right) \cos ^{2}(k / 2+\phi)\right]^{\frac{1}{2}},
\end{aligned}
$$

which give the spectrum of the whole system when all possible $k$ are taken. Moreover, the eigenstates of a nonHermitian Hamiltonian can construct a set of biorthogonal bases in association with the eigenstates of its Hermitian conjugate. For the present system, eigenstates $\left|\psi_{+}^{k}\right\rangle$, $\left|\psi_{-}^{k}\right\rangle$ of $H_{k}$ and $\left|\eta_{+}^{k}\right\rangle,\left|\eta_{-}^{k}\right\rangle$ of $H_{k}^{\dagger}$ are the biorthogonal bases of the single-particle invariant subspace, which are explicitly expressed as,

$$
\begin{gathered}
\left|\psi_{+}^{k}\right\rangle=\left(\begin{array}{c}
\cos \frac{\theta_{k}}{2} \\
\sin \frac{\theta_{k}}{2} e^{i \varphi_{k}}
\end{array}\right), \quad\left|\psi_{-}^{k}\right\rangle=\left(\begin{array}{c}
-\sin \frac{\theta_{k}}{2} \\
\cos \frac{\theta_{k}}{2} e^{i \varphi_{k}}
\end{array}\right), \\
\left|\eta_{+}^{k}\right\rangle=\left(\begin{array}{c}
\cos \frac{\theta_{k}}{2} \\
\sin \frac{\theta_{k}}{2} e^{-i \varphi_{k}}
\end{array}\right)^{*},\left|\eta_{-}^{k}\right\rangle=\left(\begin{array}{c}
-\sin \frac{\theta_{k}}{2} \\
\cos \frac{\theta_{k}}{2} e^{-i \varphi_{k}}
\end{array}\right)^{*} .
\end{gathered}
$$

It is ready to check that the biorthogonal bases $\left\{\left|\psi_{\lambda}^{k}\right\rangle,\left|\eta_{\lambda}^{k}\right\rangle\right\}(\lambda= \pm)$ obey the biorthogonal and completeness conditions

$$
\left\langle\eta_{\lambda^{\prime}}^{k^{\prime}} \mid \psi_{\lambda}^{k}\right\rangle=\delta_{\lambda \lambda^{\prime}} \delta_{k k^{\prime}}, \sum_{\lambda, k}\left|\psi_{\lambda}^{k}\right\rangle\left\langle\eta_{\lambda}^{k}\right|=1
$$

These properties are independent of the reality of the spectrum. In this paper, we focus on the case with full real spectrum. This happens when (i) $\nu=0$, the Hamiltonian goes back to a Hermitian system, (ii) $\mu=0$ and $\nu<2 \delta$. The phase diagram is true for arbitrary value of constant $\phi$, which is the basis for the subsequent investigation of the dynamics with time-dependent flux.

\section{AMPLITUDE MODULATION BY IMAGINARY BERRY PHASE}

The aim of this paper is to investigate the effect of the time-varying flux on the dynamics of the non-Hermitian system. We start with an adiabatic evolution, in which an initial eigenstate evolves into the instantaneous eigenstate of the time-dependent Hamiltonian.

From Eq. (1), we know that $H$ is a periodic function of $\phi$ with $H(\phi)=H(\phi+2 \pi)$ and $H_{k}(\phi)=H_{k}(\phi+2 \pi)$. Considering the time-dependent flux $\phi(t)$, any eigenstate $\left|\psi_{\lambda}^{k}(0)\right\rangle$ will return back to $\left|\psi_{\lambda}^{k}(0)\right\rangle$ if $\phi(t)$ varies adiabatically from 0 to $2 \pi$, and the evolved state is the instantaneous eigenstate $\left|\psi_{\lambda}^{k}(\phi)\right\rangle$. More explicitly, we regard the flux as a linear function of time, that is, $\phi=\beta t$. And the adiabatic evolution of the initial eigenstate $\left|\psi_{\lambda}^{k}(0)\right\rangle$ under the time-dependent Hamiltonian $H(\phi(t))$ can be expressed as

$$
\begin{aligned}
\left|\Psi_{\lambda}^{k}(\phi)\right\rangle & =\mathcal{T} \exp \left[-i \int_{0}^{t} H(t) \mathrm{d} t\right]\left|\psi_{\lambda}^{k}(0)\right\rangle \\
& =e^{i\left(\alpha_{k}^{\lambda}+\gamma_{k}^{\lambda}\right)}\left|\psi_{\lambda}^{k}(\phi)\right\rangle .
\end{aligned}
$$

Here the dynamics phase defined by $\alpha_{k}^{\lambda}(\phi)$ and adiabatic phase $\gamma_{k}^{\lambda}(\phi)$ have the form

$$
\begin{aligned}
\alpha_{k}^{\lambda}(\phi) & =-\frac{1}{\beta} \int_{0}^{\phi} \varepsilon_{\lambda}^{k}(\phi) \mathrm{d} \phi \\
\gamma_{k}^{\lambda}(\phi) & =i \int_{0}^{\phi}\left\langle\eta_{\lambda}^{k}(\phi)\left|\partial_{\phi}\right| \psi_{\lambda}^{k}(\phi)\right\rangle \mathrm{d} \phi \\
& =-\int_{0}^{\phi} \frac{2 \delta J^{2} \mathrm{~d} \phi}{\varepsilon_{\lambda}^{k}\left[\varepsilon_{\lambda}^{k}-J(\mu+i \nu)\right]} .
\end{aligned}
$$

In this paper, we focus on the system with full real spectrum. Then the dynamics phase depends only on the instantaneous dispersion relation and is always real. We have an interest in the adiabatic phase since it may have an extra contribution to an evolved state. In the following, we will present several features of $\gamma_{k}^{\lambda}(\phi)$, based on Eq. (22).

It is obvious that we have $\gamma_{k}^{\lambda}(\phi)=0$, if the dimerization factor $\delta=0$, which is a necessary condition for nonzero adiabatic phase. In contrast, we have nonzero $\gamma_{k}^{\lambda}(\phi)$ in the case of zero staggered potential, which indicates the significance of the Peierls distortion to the adiabatic phase. It is easy to check that

$$
\frac{\partial^{2}}{\partial^{2} \phi} \gamma_{k}^{\lambda}(\phi) \propto \sin (k+2 \phi)
$$



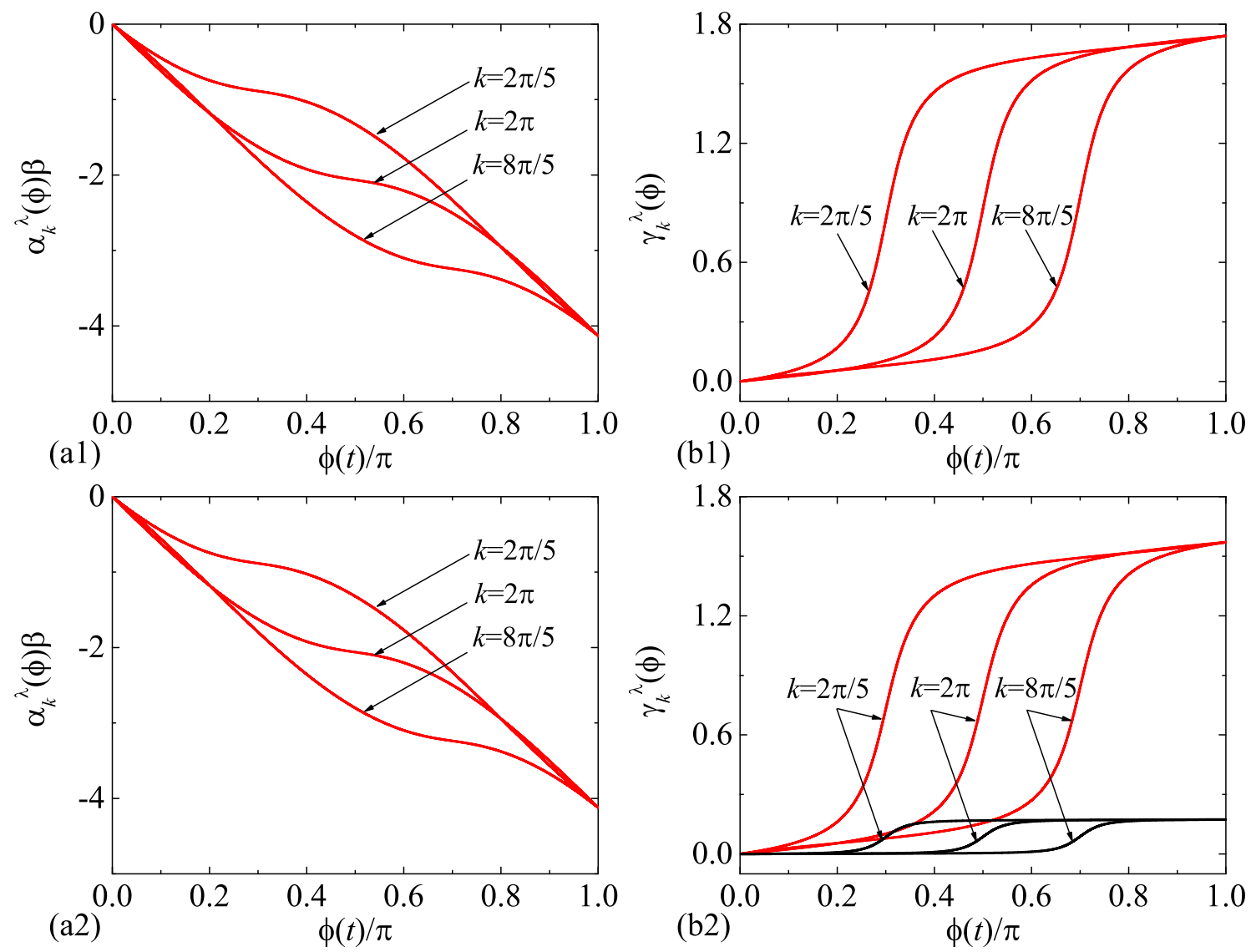

FIG. 2. (Color online) Plots of the reduced dynamic phase $\alpha_{k}^{\lambda} \beta$ and the geometric phase $\gamma_{k}^{\lambda}$ as functions of $\phi$, expressed in Eq. (21) and (22), for different values of $k$ in a typical (a) Hermitian system with $\delta=-0.15, \mu=0.05$, and $\nu=0$; (b) non-Hermitian system with $\delta=-0.15, \mu=0$, and $\nu=0.05$. Both systems have full real spectrum. The real (imaginary) parts of the phases are plotted in red (black). (a) It is shown that both phases are real for the Hermitian system, while the adiabatic phase is complex for the non-Hermitian system. The dynamic phases and the real part of adiabatic phases in both cases have a slight difference. The shapes of all the phases are in agreement with the approximate expressions in Eqs. (33), (34), (37), and (38). Here the reduced dynamic phase $\alpha_{k}^{\lambda} \beta$ and the geometric phase $\gamma_{k}^{\lambda}$ are expressed in units of radian.

which leads to $\partial_{\phi}^{2} \gamma_{k}^{\lambda}(\phi)=0$ at $\phi_{c}=\pi / 2-k / 2$. This fact indicates that both the real and imaginary parts of $\gamma_{k}^{\lambda}(\phi)$ experience a maximal (or minimal) change at this point. Furthermore, including Hermitian and nonHermitian systems, we note that

$$
\frac{\partial \gamma_{k}^{\lambda}(n \pi)}{\partial k}=\frac{\partial \alpha_{k}^{\lambda}(n \pi)}{\partial k}=0
$$

In view of the fact that for an arbitrary function $g(\cos (k+2 \phi))$, one can always obtain

$$
\begin{aligned}
& \frac{\partial}{\partial k} \int_{0}^{n \pi} g(\cos (k+2 \phi)) \mathrm{d} \phi \\
= & \frac{1}{2} g(\cos (k+2 \phi))_{0}^{n \pi}=0 .
\end{aligned}
$$

This shows that the dynamic and Berry phases are $k$ independent after $\phi$ varying $n \pi$, which ensures that any arbitrary initial state involved in the upper or lower band solely can revive back exactly after $\phi$ varying $2 \pi$.

For a Hermitian system, the adiabatic phase is always real, which ensures the probability preserving evolution.
While, the probability of an evolved state changes due to the imaginary part of the adiabatic phase in a nonHermitian system. The gain or loss of probability depends on the sign of the imaginary phase. There are several rigorous results for the phase.

For given parameters $\{\delta, \mu, \nu\}$, the adiabatic phase of an eigenstate in $\lambda$ band obeys the identity

$$
\gamma_{k}^{\lambda}(n \pi)=-\gamma_{k}^{\lambda}(-n \pi)
$$

which is obtained from Eq. (22), owing to the fact

$$
\varepsilon_{\lambda}^{k}(\phi-n \pi)=\varepsilon_{\lambda}^{k}(\phi) .
$$

This means that the direction of $\phi$ can determine the sign of $\operatorname{Im} \gamma_{k}^{\lambda}(n \pi)$, controlling the probability of an evolved state.

In addition, the sign of $\operatorname{Re} \gamma_{k}^{\lambda}(\phi)$ and $\operatorname{Im} \gamma_{k}^{\lambda}(\phi)$ could also depend on the sign of $\delta$ and $\nu$ via the Eqs. (28) and 
(29), that is,

$$
\begin{gathered}
\operatorname{Re} \gamma_{k}^{\lambda}(\phi)=\operatorname{sgn}(\delta) \int_{0}^{\phi} \frac{-2|\delta| J^{2} \mathrm{~d} \phi}{\left[\left(B_{k}\right)^{2}+J^{2} \nu^{2}\right]}, \\
\operatorname{Im} \gamma_{k}^{\lambda}(\phi)=\operatorname{sgn}(\delta \nu \lambda) \int_{0}^{\phi} \frac{-2 J^{3}|\delta \nu| \mathrm{d} \phi}{B_{k}\left[\left(B_{k}\right)^{2}+J^{2} \nu^{2}\right]},
\end{gathered}
$$

which yield

$$
\begin{aligned}
& {\left[\gamma_{k}^{\lambda}(\phi)\right]_{\delta}=-\left[\gamma_{k}^{\lambda}(\phi)\right]_{-\delta},} \\
& {\left[\gamma_{k}^{\lambda}(\phi)\right]_{\nu}=\left[\gamma_{k}^{\lambda}(\phi)\right]_{-\nu}^{*} .}
\end{aligned}
$$

Together with the Eq. (26), we show that the sign of $\operatorname{Im}\left(\gamma_{k}^{\lambda}\right)$ is determined by the following expression that

$$
\operatorname{sgn}\left[\operatorname{Im} \gamma_{k}^{\lambda}\left(\lambda^{\prime} n \pi\right)\right]=-\operatorname{sgn}\left(\nu \delta \lambda \lambda^{\prime}\right) \quad\left(\lambda^{\prime}= \pm\right),
$$

which directly results in the amplification or attenuation of an evolved state.

Besides the exact results, it is useful to arrive at the whole profile of phases as a function of a group of parameters $\{\phi, k, \delta, \mu, \nu, \lambda\}$. The approximate expressions of the phases for Hermitian and non-Hermitian systems could be obtained from straightforward derivations as following, respectively.

For a Hermitian system $(\nu=0)$, we have

$$
\begin{aligned}
& \gamma_{k}^{\lambda}(\phi) \approx \frac{\operatorname{sgn}(\delta / 2)}{\sqrt{1-\delta^{2}}}\left\{\tan ^{-1} \Theta_{k}(0)-\tan ^{-1} \Theta_{k}(\phi)\right. \\
& \left.-\operatorname{sgn}(\mu \lambda)\left[\tan ^{-1}\left(|\mu| \Gamma_{k}(\phi)\right)-\tan ^{-1}\left(|\mu| \Gamma_{k}(0)\right)\right]\right\},
\end{aligned}
$$

and

$$
\begin{aligned}
& \alpha_{k}^{\lambda}(\phi) \approx \frac{\lambda}{4 \beta}\left\{B_{k}(0)(k-\pi)-B_{k}(\phi)(k+2 \phi-\pi)\right. \\
& \left.+\frac{J\left(4 \delta^{2}+\mu^{2}\right)}{\sqrt{1-\delta^{2}}} \ln \frac{B_{k}(\phi)-2|\delta| J \Theta_{k}(\phi)}{B_{k}(0)-2|\delta| J \Theta_{k}(0)}\right\},
\end{aligned}
$$

where

$$
\begin{aligned}
\Theta_{k}(\phi) & =\sqrt{\delta^{-2}-1}(k+2 \phi-\pi) / 2, \\
\Gamma_{k}(\phi) & =J \Theta_{k}(\phi) / B_{k}(\phi),
\end{aligned}
$$

are even functions of $\delta, \mu$, and $\lambda$. It is shown that $\gamma_{k}^{\lambda}(\phi)$ is a real number, preserving the probability.

While, for the non-Hermitian system $(\mu=0, \nu<2 \delta)$, we have

$$
\begin{aligned}
& \gamma_{k}^{\lambda}(\phi) \approx \frac{\operatorname{sgn}(\delta / 2)}{\sqrt{1-\delta^{2}}}\left\{\tan ^{-1} \Theta_{k}(0)-\tan ^{-1} \Theta_{k}(\phi)\right. \\
& \left.-i \operatorname{sgn}(\nu \lambda)\left[\tanh ^{-1}\left(|\nu| \Gamma_{k}(\phi)\right)-\tanh ^{-1}\left(|\nu| \Gamma_{k}(0)\right)\right]\right\}
\end{aligned}
$$

and

$$
\begin{aligned}
& \alpha_{k}^{\lambda}(\phi) \approx \frac{\lambda}{4 \beta}\left\{B_{k}(0)(k-\pi)-B_{k}(\phi)(k+2 \phi-\pi)\right. \\
& \left.+\frac{J\left(4 \delta^{2}-\nu^{2}\right)}{\sqrt{1-\delta^{2}}} \ln \frac{B_{k}(\phi)-2|\delta| J \Theta_{k}(\phi)}{B_{k}(0)-2|\delta| J \Theta_{k}(0)}\right\},
\end{aligned}
$$
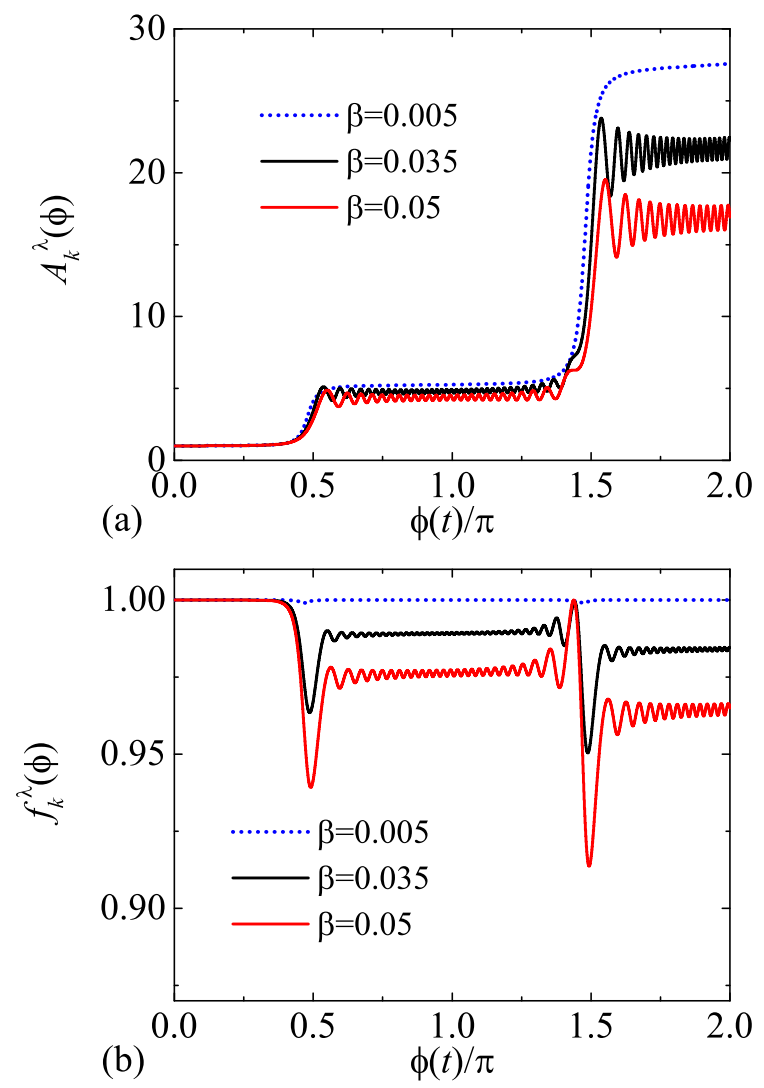

FIG. 3. (Color online) Numerical simulations for the time evolutions with different $\beta$. Amplification factor $A_{k}^{\lambda}(\phi)$ and fidelity $f_{k}^{\lambda}(\phi)$ for the initial state with $k=\pi / 25$ and $\lambda=$ - are plotted for the system with $\delta=0.15, \mu=0, \nu=$ -0.2 , and $N=50$. We can see the evolution results are close to that, $A_{\pi / 25}^{-}(\pi)=27.58, f_{\pi / 25}^{-}(\pi)=1$, obtained in the adiabatic limit. This indicates that the amplitude control could be realized with a high fidelity via a quasi-adiabatic process.

where $\Theta_{k}(\phi)$ and $\Gamma_{k}(\phi)$ have the same form as above, but even functions of $\delta, \nu$, and $\lambda$. The remarkable feature of $\gamma_{k}^{\lambda}$ is that it is a complex number. The approximate expression shows that both real and imaginary parts of $\gamma_{k}^{\lambda}(\phi)$ are flat functions of $\phi$ except for the region around the point $\phi_{c}$, in which they experience a drastic change. The key feature of an imaginary phase is its sign, which affects the amplitude of the evolved eigenstate directly, determining the gain or loss of the probability.

To verify and demonstrate the above analysis, numerical simulations are performed to investigate the dynamics behavior of a quasi-adiabatic process. We compute the time evolution of an eigenstate by using a uniform mesh in the time discretization for the time-dependent Hamiltonian $H(t)$. The amplification factor (gain) is defined as

$$
\left.\left.A_{k}^{\lambda}(\phi)=\| \psi_{\lambda}^{k}(0)\right\rangle\left.\right|^{-1}\left|\mathcal{T} \exp \left[-i \int_{0}^{t} H(t) \mathrm{d} t\right]\right| \psi_{\lambda}^{k}(0)\right\rangle \mid,
$$




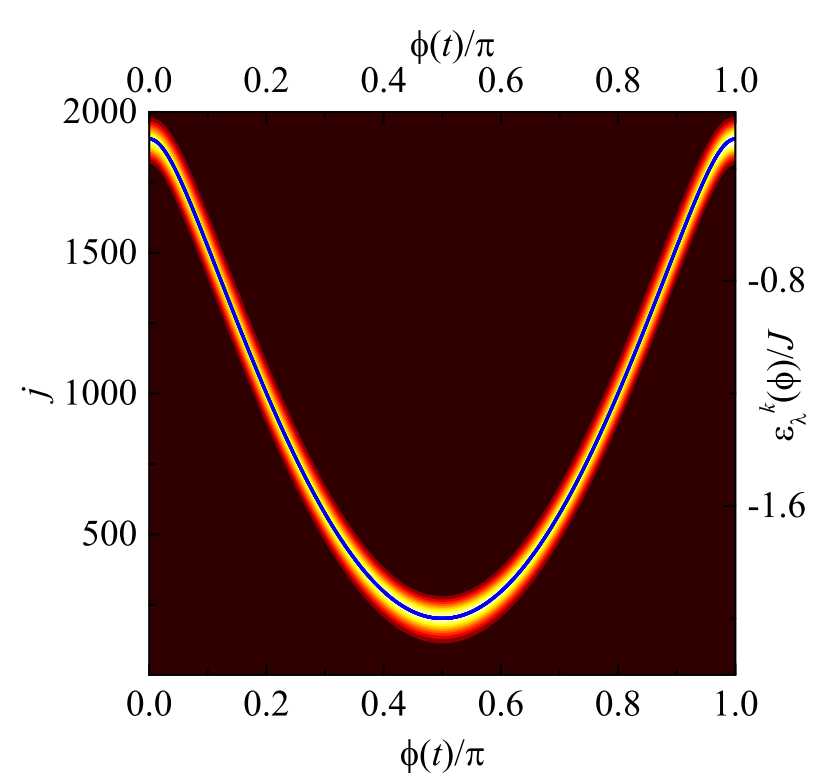

FIG. 4. (Color online) The comparison between the dispersion relation (blue dot) and the trajectory of the center of the wave packet (color contour map). The simulation is computed as follows: the wave packet with $k_{0}=\pi / 2, \alpha=0.05$, and $N_{\mathrm{A}}=1900$, in the lower band of the system with $\delta=-0.15$, $\mu=0, \nu=0.05$, and $N=1000$, subjected in the external field with $\beta=0.001$. This shows that the two are in close proximity owing to the fact that the contribution of the geometric phase to the wave packet position becomes negligible when compared with the dynamic phase. Only the imaginary part of the geometric phase takes an important role in the dynamics of the wave packet.

which is the ratio of the output magnitude to the input magnitude of an eigenstate. We use the fidelity $f_{k}^{\lambda}(\phi)$, which is defined as

$$
f_{k}^{\lambda}(\phi)=\left|\left\langle\Psi_{\lambda}^{k}(\phi)\left|\mathcal{T} \exp \left[-i \int_{0}^{t} H(t) \mathrm{d} t\right]\right| \psi_{\lambda}^{k}(0)\right\rangle\right|,
$$

to describe the derivation between adiabatic and quasiadiabatic processes. For an adiabatic process $(\beta \rightarrow 0)$, we have $A_{k}^{\lambda}(\phi)=\exp \left[-\operatorname{Im}\left(\gamma_{k}^{\lambda}(\phi)\right)\right]$ and $f_{k}^{\lambda}(\phi)=1$. We can employ $A_{k}^{\lambda}(\pi)$ to describe the amplification factor for an arbitrary quantum state. The computation is performed by using a uniform mesh in the time discretization for the time-dependent Hamiltonian $H(t)$. As an example, in Fig. 3, we show the evolution of $A_{k}^{\lambda}(\phi)$ and $f_{k}^{\lambda}(\phi)$ for different values of $\beta$. The plot in (a) and (b) shows the quasi-adiabatic process can be close to the adiabatic one.

\section{WAVE PACKET DYNAMICS}

Before starting the investigation of the wave packet dynamics in the present system, we would like to give a brief review on the dynamics in a uniform ring. In the case of $\delta=\mu=\nu=0$, it has been shown that the dynamics of a wave packet is the same as that driven by a linear field with strength $\beta$, according to the quantum Faraday's law [39]. Furthermore, it is turned out that the center path of a wave packet driven by a linear field accords with the dispersion of the Hamiltonian in the absence of the field within the adiabatic regime [40].

$$
x_{c}(\phi)=x_{c}(0)+\frac{1}{\beta}\left\{\varepsilon^{k_{c}}(\phi)-\varepsilon^{k_{c}}(0)\right\},
$$

where $\varepsilon^{k}(\phi)$ is the dispersion relation and $k_{c}$ is the central momentum of the wave packet.

Now, we switch gears to the case of the present model. We note that the geometrical phase takes a role in the dynamics and the dynamics of the wave packet cannot be simply understood in terms of a semiclassical picture [41 43]. Notice that the trajectory of a wave packet is essentially not only determined by the dispersion relation for the field-free system but also by the geometric phase. In other words, the dependence of the geometric phase on $k$ should be taken into account.

However, in the adiabatic limit, $\beta$ is very small. The contribution of the geometric phase to the trajectory becomes negligible, that is,

$$
\operatorname{Re} \gamma_{k}^{\lambda}(\phi) \ll \alpha_{k}^{\lambda}(\phi) .
$$

The main effect of the geometric phase on the dynamics of the wave packet is then just the modulation of the amplitude.

To verify and demonstrate the above analysis, numerical simulations are performed to investigate the dynamics behavior. We compute the time evolution of the wave packet by the same method as mentioned above. The initial Gaussian wave packet has the form

$$
\left|G_{k_{0}}^{N_{\mathrm{A}}}(0)\right\rangle=\frac{1}{\sqrt{\Omega_{1}}} \sum_{j=1}^{2 N} e^{-\frac{\alpha^{2}}{2}\left(j-N_{\mathrm{A}}\right)^{2}} e^{i k_{0} j}|j\rangle
$$

with the velocity $k_{0} \in[0,2 \pi]$, centered at the $N_{\mathrm{A}}$ th site, where $|j\rangle=c_{j}^{\dagger}|0\rangle$ and $\Omega_{1}$ is a normalization factor.

For one thing, we consider the trajectories of the wave packet with different $\beta$, as comparison to the dispersion relation. From the plots in Fig. 4, we find that for small $\beta$, the trajectory accords with the dispersion well, while as $\beta$ increases, the deviation becomes obvious.

For another thing, we investigate the flux-controlled probability of the wave packet. It can be rewritten in the form

$$
\left|G_{k_{0}}^{N_{\mathrm{A}}}(0)\right\rangle=\sum_{k}\left(g_{+}^{k}\left|\psi_{+}^{k}\right\rangle+g_{-}^{k}\left|\psi_{-}^{k}\right\rangle\right) .
$$

Here, we do not give the explicit expression of the coefficient $g_{\lambda}^{k}$, since the following analysis is independent of $g_{\lambda}^{k}$. Through an adiabatic evolution, we have

$$
\left|G_{k_{0}}^{N_{\mathrm{A}}}(2 \pi n / \beta)\right\rangle=\sum_{k}\left(e^{i \Omega_{+}} e^{\zeta} g_{+}^{k}\left|\psi_{+}^{k}\right\rangle+e^{i \Omega_{-}} e^{-\zeta} g_{-}^{k}\left|\psi_{-}^{k}\right\rangle\right),
$$




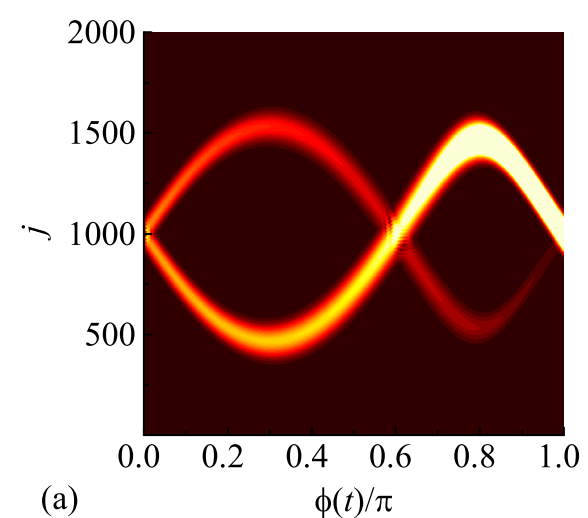

(a) $\phi(t) / \pi$
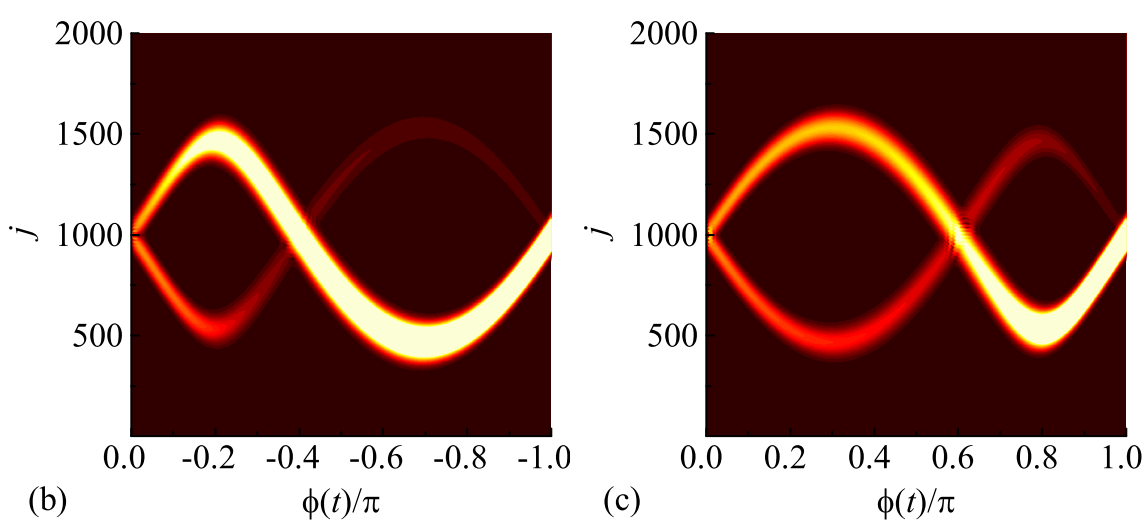

FIG. 5. (Color online) The profiles of the time evolution of a wave packet in several typical cases. The initial wave packet is in the form of Eq. (43) with $k_{0}=1.4 \pi, \alpha=0.05$, and $N_{\mathrm{A}}=1000$, and the external field varies with $\beta=0.001$. (a) In the case of $\delta=-0.7, \mu=0$, and $\nu=1.3$, the plot is shown that the probability of the sub-wave packet in one band increases but the one in another band decreases. In the cases of (b) and (c), we take the same parameters with (a) but opposite $\phi$ and $\delta$, respectively. Comparing with the profile in (a), we can see the completely opposite behaviors in (b) and (c). This is in agreement with our analytical prediction.
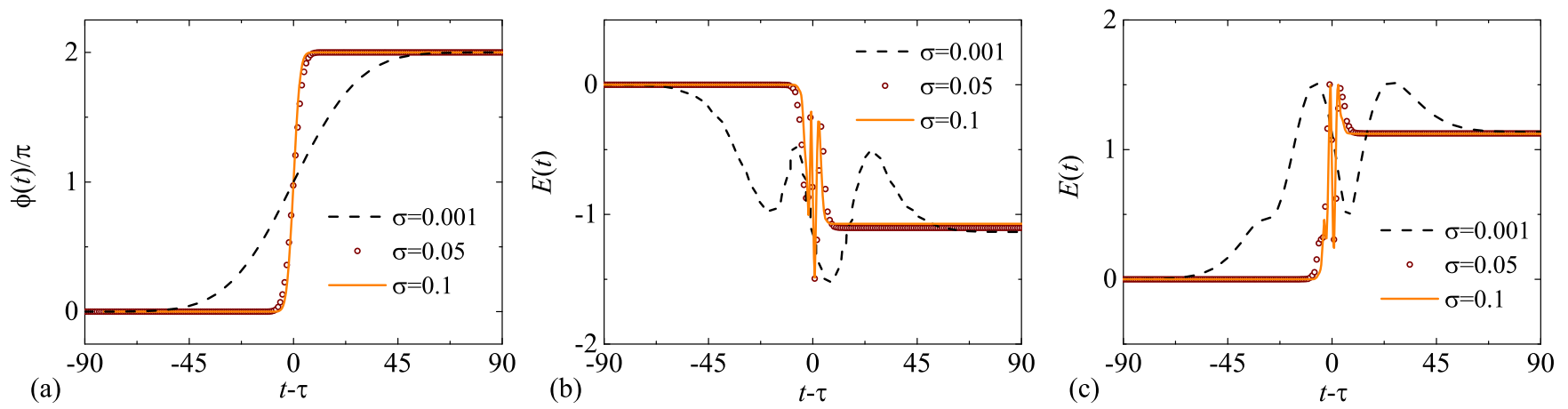

FIG. 6. (Color online) Profiles of the reduced energy defined in Eq. (48) of an evolved wave packet in Eq. (43) is controlled by a Gaussian-type time-varying field in Eq. (47). The parameters of the wave packet and system are $k_{0}=1.5 \pi, \delta=-0.7, \mu=0$, and $\nu=1.3$. (a) The shapes of the field as a function of time in Eq. (47) with different $\sigma$. (b) Plots of $E(t)$ of the field in (a). (c) Same as (b) but with the opposite flux. The simulation result is in agreement with the result $E(\infty)=-1.14$ for (b) and 1.14 for (c), obtained in the adiabatical limit. This indicates that the amplitude of the wave packet can be well controlled via a quasi-adiabatic process in a short time. Here the time $t-\tau$ and the reduced energy $E(t)$ are expressed in units of $1 / J$ and $J$, respectively.

where $\Omega_{ \pm}$is a real number, and $\zeta=-\operatorname{Im} \gamma_{k}^{+}(2 \pi n)$. In the case of $\lambda \zeta \gg 1$, we have

$$
\left|G_{k_{0}}^{N_{\mathrm{A}}}(2 \pi n / \beta)\right\rangle \approx e^{i \Omega_{\lambda}} e^{\lambda \zeta} \sum_{k} g_{\lambda}^{k}\left|\psi_{\lambda}^{k}\right\rangle .
$$

It is shown that only the component in either upper or lower sub-band survives. It also seems that the final state collapses to one of two sub-bands in the context of Dirac probability. The sign of $\zeta$ is crucial for the direction of the collapse. We compute the evolution for two cases with opposite flux. The result plotted in Fig. 5] shows that the evolved wave packet in upper or lower sub-band survives for two different varying fluxes, which is in agreement with our prediction.

In practice, the flux control can be implemented by a pulsed flux. We simulate this process by a Gaussian- shaped flux with the form

$$
\phi(t)=2 \sqrt{\sigma \pi} \int_{0}^{t} e^{-\sigma(t-\tau)^{2}} \mathrm{~d} t
$$

which contributes $2 \pi$ flux during the process. To characterize the feature, we introduce the reduced energy

$$
E(t)=\frac{\sum_{k, \lambda} \varepsilon_{\lambda}^{k}\left|\left\langle\eta_{\lambda}^{k} \mid G_{k_{0}}^{N_{\mathrm{A}}}(t)\right\rangle\right|^{2}}{\sum_{k, \lambda}\left|\left\langle\eta_{\lambda}^{k} \mid G_{k_{0}}^{N_{\mathrm{A}}}(t)\right\rangle\right|^{2}} .
$$

And in the adiabatic limit, we have $E(\infty) \approx \varepsilon_{+}^{k_{0}}$ or $\varepsilon_{-}^{k_{0}}$, i.e., it converges to a positive (negative) constant if the upper (lower) sub-wave packet survives. Alternatively, we can replace $\varepsilon_{\lambda}^{k}$ by $\lambda$ in Eq. (48) to redefine $E(t)$, which leads to $E(\infty) \approx+1$ or -1 even for a non-adiabatic 
process. Here we take the former, because $E(\infty)$ can indicate the deviation between the adiabatic and nonadiabatic processes. In Fig. 6] results are plotted for different values of $\sigma$, which determine the rate of the flux change. These results clearly demonstrate the finding of this paper that the external field can be utilized to control the quantum state on demand via a quasi-adiabatic process within a relatively short time.

\section{SUMMARY}

In this paper, the particle dynamics of the nonHermitian Rice-Mele model driven by a time-dependent external field has been theoretically investigated. The analysis shows that the Berry phase can be a complex number in the non-Hermitian regime. This results in the amplification and attenuation of the amplitude of an evolved quantum state. We found that it can have full real spectrum for any constant field, and the Berry phase with respect to a varying field has a constant imaginary part for an arbitrary initial state either in the upper or lower energy sub-band. The dependence of the imaginary part of the Berry phase on the parameters, such as lattice distortion, imaginary potential, and the direction of the flux, was explicitly presented. Numerical simulation indicates that the amplitude control of a wave packet can be accomplished by a quasi-adiabatic process within a relatively short time.

\section{ACKNOWLEDGMENTS}

We acknowledge the support of the National Basic Research Program (973 Program) of China under Grant No. 2012CB921900 and CNSF (Grant No. 11374163).

\section{REFERENCES}

[1] S. Pancharatnam, Proc. Indian Acad. Sci. A 44247 (1956).

[2] M. V. Berry, Proc. R. Soc. London A 392, 45 (1984).

[3] D. Xiao, M. -C. Chang, and Q. Niu, Rev. Mod. Phys. 82, 1959 (2010).

[4] J. C. Garrison and E. M. Wright, Phys. Lett. A 128, 177 (1988).

[5] G. Dattoli, R.Mignani, and A. Torre, J. Phys. A 23, 5795 (1990).

[6] C. Z. Ning and H. Haken, Phys. Rev. Lett. 68, 2109 (1992).

[7] D. J. Moore and G. E. Stedman, Phys. Rev. A 45, 513 (1992).

[8] Xiao-Chun Gao, Jing-Bo Xu, and Tie-Zheng Qian, Phys. Rev. A 46, 3626 (1992).

[9] Marcel Pont, R. M. Potvliege, Robin Shakeshaft, and Philip H. G. Smith, Phys. Rev. A 46, 555 (1992).

[10] S. Massar, Phys. Rev. A 54, 4770 (1996).

[11] Y. C. Ge and M. S. Child, Phys. Rev. A 58, 872 (1998).

[12] Robert S. Whitney, Yuriy Makhlin, Alexander Shnirman, and Yuval Gefen, Phys. Rev. Lett. 94, 070407 (2005).

[13] H. Mehri-Dehnavi and A. Mostafazadeh, J. Math. Phys. 49, 082105 (2008).

[14] Alexander I. Nesterov and S. G. Ovchinnikov, Phys. Rev. E 78, 015202(R) (2008).

[15] Xiao-Dong Cui and Yujun Zheng, Phys. Rev. A 86, 064104 (2012).

[16] Shi-Dong Liang and Guang-Yao Huang, Phys. Rev. A 87, 012118 (2013).

[17] C. Li, G. Zhang, X. Z. Zhang, and Z. Song, Phys. Rev. A 90, 012103 (2014).

[18] C. M. Bender and S. Boettcher, Phys. Rev. Lett. 80, 5243 (1998).

[19] C. M. Bender, D. C. Brody, H. F. Jones, and B. K. Meister, Phys. Rev. Lett. 98, 040403 (2007).
[20] A. Mostafazadeh, Phys. Rev. Lett. 102, 220402 (2009); Phys. Rev. A 80, 032711 (2009).

[21] S. Longhi, Phys. Rev. B 80, 165125 (2009).

[22] X. Z. Zhang, L. Jin, and Z. Song, Phys. Rev. A 87, 042118 (2013).

[23] X. Q. Li, X. Z. Zhang, G. Zhang, and Z. Song, arXiv:1409.0420

[24] G. Zhang, X. Q. Li, X. Z. Zhang, and Z. Song, arXiv:1412.7630

[25] T. E. Lee, F. Reiter, and N. Moiseyev, Phys. Rev. Lett. 113, 250401 (2014).

[26] S. Longhi, Phys. Rev. A 88, 062112 (2013)

[27] W. H. Hu, L. Jin, Y. Li, and Z. Song, Phys. Rev. A 86, 042110 (2012).

[28] Ingrid Rotter, J. Phys. A: Math. Theor. 42153001 (2009).

[29] M. J. Rice and E. J. Mele, Phys. Rev. Lett. 49, 1455 (1982).

[30] J. Dalibard, Y. Castin, and K. Mølmer, Phys. Rev. Lett. 68, 580 (1992).

[31] K. Mølmer, Y. Castin, and J. Dalibard, J. Opt. Soc. Am. B 10, 524 (1993).

[32] R. Dum, P. Zoller, and H. Ritsch, Phys. Rev. A 45, 4879 (1992).

[33] M. B. Plenio and P. L. Knight, Rev. Mod. Phys. 70, 101 (1998).

[34] A. J. Daley, Adv. Phys. 63, 77 (2014).

[35] S. Tung, V. Schweikhard, and E. A. Cornell, Phys. Rev. Lett. 97, 240402 (2006).

[36] R. A. Williams, S. Al-Assam, and C. J. Foot, Phys. Rev. Lett. 104, 050404 (2010).

[37] T. J. Osborne and N. Linden, Phys. Rev. A 69, 052315 (2004).

[38] S. Yang, Z. Song, and C.P. Sun, Phys. Rev. A 73, 022317 (2006). 
[39] W. H. Hu, L. Jin, and Z. Song, Quantum Inf. Process 12(11), 3569 (2013).

[40] S. Lin, X. Z. Zhang, and Z. Song, Phys. Rev. A 90, 063411 (2014).
[41] F. Bloch, Z. Phys. 52, 555 (1929).

[42] N. W. Ashcroft and N. D. Mermin, Solid State Physics (Harcourt, Fort Worth, TX, 1976), Chap. 12.

[43] C. Kittel, Quantum Theory of Solids, 2nd ed. (Wiley, New York, 1987). 